\title{
Determinación de Propiedades Termofísicas de Alimentos en Función de la Concentración y la Temperatura empleando un Programa Computacional
}

\author{
Armando Alvis ${ }^{\left(1^{\star}\right)}$, Isaac Caicedo ${ }^{(2)}$, Pierre Peña ${ }^{(2)}$ \\ (1) Universidad de Córdoba, Facultad de Ingeniería, Depto. de Ingeniería de Alimentos, \\ Carrera 6 No 76-103, Córdoba-Colombia (e-mail: aalvis2@hotmail.com) \\ (2) Universidad de Córdoba, Facultad de Ingeniería, Depto. de Ingeniería de Sistemas, \\ Carrera 6 No 76-103, Córdoba-Colombia
}

Recibido Mar. 31, 2011; Aceptado May. 26, 2011; Versión Final recibida Sep. 01, 2011

\begin{abstract}
Resumen
Se presenta el desarrollo de un programa computacional que permite calcular las propiedades termofísicas de un alimento fresco o procesado en función de la composición del alimento y la temperatura. La herramienta, denominada DEPROTER (Determinación de Propiedades Termofísicas) permite determinar el calor específico, la conductividad térmica, la difusividad térmica y la densidad. Para validar el modelo se usaron datos experimentales encontrados en la literatura de muestras de ñame frescas y procesadas, utilizando diferentes temperaturas de proceso. Los datos fueron procesados automáticamente por el software, para la generación de los valores de las propiedades térmicas con desviaciones pequeñas con respecto a los datos experimentales.
\end{abstract}

Palabras clave: propiedades de alimentos, calor específico, densidad, conductividad térmica, difusividad térmica

\section{Determination of Thermal Properties of Foods as Function of Concentration and Temperature using a Computer Program}

\begin{abstract}
The development of a computer program to calculate thermophysical properties of fresh or processed food as function of food concentration and temperature is presented. The computer tool named DEPROT (Determination of Thermophysical Properties) allows calculating the specific heat, thermal conductivity, thermal diffusivity and density. To validate the model, experimental data from the literature for fresh and processed samples of yam were used. The data were processed by heat treatment, using different process temperatures. The experimental data were automatically processed by the software to generate the values of the thermal properties with low deviations with respect to the experimental data.
\end{abstract}

Keywords: food properties, specific heat, density, thermal conductivity, thermal diffusivity 


\section{INTRODUCCION}

Los modelos matemáticos proporcionan una de las herramientas básicas para describir procesos físicos y para explicar y predecir el comportamiento en condiciones variadas. Recientemente, la informática ha estimulado un mayor interés en la elaboración de modelos matemáticos de transferencia de calor en los procesos de alimentos como (esterilización, congelación, refrigeración, escaldado y fritura) (Schwartzberg y Chao, 1982; Califano y Cálvelo, 1983; Moreira et al., 1995; Pelegrina et al., 2000, Bon et al., 2001; Singh y Erdogdu, 2004 y Alvis et al., 2010). El modelo escogido para determinar las propiedades termofísicas en fresco o durante el proceso térmico fue seleccionado por simplicidad, ya que involucra la composición del producto y la temperatura (Choi y Okos, 1985).

Las propiedades térmicas (calor específico, conductividad térmica y difusividad térmica influyen en la transferencia de calor. Su conocimiento es necesario para el cálculo y diseño de procesos y equipos y en la calidad del producto; están influenciadas en un mayor o menor grado por la temperatura y la composición del producto, durante un tratamiento térmico (Rahman, 1995; Singh, 2000; Alvis et al., 2009). Las propiedades térmicas de frutas y vegetales son necesarias para calcular la rapidez de calentamiento o enfriamiento en procesos o para estimar las cantidades de calor requeridas en los procesos como: escaldado, pasteurización, evaporación, fritura, refrigeración, congelación, esterilización, secado entre otra, en los cuales hay intercambio de energía y masa (Alvis et al., 2009).

Un proceso de simulación utiliza avanzados modelos matemáticos para predecir los cambios físicos, químicos o microbiológicos, que han sido ampliamente validados con datos experimentales encontrados en la literatura. Por lo tanto, las predicciones de los procesos de alimentos se consideran altamente confiables (Rahman, 1995; Barbosa-Canovas et al., 1997; Singh y Erdogdu, 2004; Alvis et al., 2010). El modelo propuesto se basa en los modelos matemáticos encontrados por Choi y Okos (1985) y se utilizaran en el software para determinar las propiedades termofísicas a partir de los modelos matemáticos siguientes ecuaciones (1) a (4)..

$$
\begin{aligned}
& c p=\sum_{i=1}^{n} C p_{i} X_{i} \\
& k=\sum_{i=1}^{n} K_{i} X_{i} \\
& \alpha=\sum_{i=1}^{n} \alpha_{i} X_{i} \\
& p=\frac{1}{\sum_{i=1}^{n} \frac{X_{i}}{\rho_{i}}}
\end{aligned}
$$

En las ecuaciones (1) a (4) el subíndice i se refiere al componente considerado, humedad, grasa, proteína, ceniza, fibra, carbohidratos, hielo:

El presente trabajo describe un software elaborado por los autores cuyo nombre es DEPROT (Determinación de Propiedades Termofísicas) para la estimación del calor específico, difusividad y conductividad térmica y densidad en diversas operaciones a fin de que los procesos puedan ser optimizados y automatizados a partir de un punto de vista de ingeniería conforme al modelo propuesto. 


\section{MATERIALES Y MÉTODOS}

Respecto a las herramientas de construcción de software, el programa de computadoras se escribió en el lenguaje de programación orientado a objetos llamado Java, utilizando el entorno de desarrollo denominado NetBean (en su versión 6) y la interfaz de usuario se implementó con la interfaz de programación conocida con el nombre de Swing. En lo concerniente a la metodología de desarrollo de software se utilizó el modelo en cascada, cuya etapa inicial consiste en la recolección de requisitos funcionales que se vislumbran en el diagrama de casos de uso de la figura 1. Con base en dicha especificación, se realizó un modelo cuya vista estática la describe en un diagrama de clases (ver en la figura 2 la clase principal del aplicativo), mientras que la vista dinámica la componen los diagramas de secuencia y de estados de las figuras 3 y 4 respectivamente.

Después del modelado de la aplicación, se codificó el programa de computadoras de acuerdo a las ecuaciones matemáticas previamente expuestas, las cuales corresponden con los métodos de la clase denominada ComputosPropiedadesTermicas, de tal manera, que el funcionamiento de la aplicación se simplifica a una interfaz de usuario gráfica, implementada en la clase PropiedadesTermicasGUI, la cual invoca los métodos de la clase ComputosPropiedadesTermicas (ver figura 3). En resumen, la aplicación pasa a través de los estados de la figura 4, iniciando con la captura de datos, siguiendo con su procesamiento y concluye con la muestra de los datos resultantes, como se aprecia en la figura 5 donde se muestra la interfaz gráfica de usuario de la aplicación.

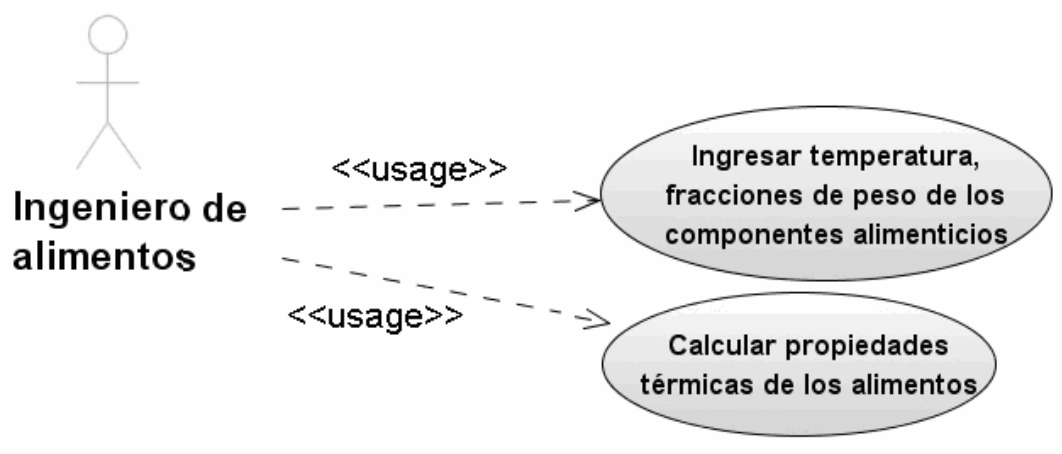

Fig. 1: Diagrama de casos de uso

Con la interfaz en mención, el usuario puede ingresar, en la parte superior de la ventana, la fracción de peso de cada componente de un alimento específico y la temperatura que se tiene en consideración; posteriormente, al pinchar el botón etiquetado como "Calcular propiedades térmicas", la aplicación muestra en la parte inferior los datos resultantes de calcular la conductividad y difusividad térmica, la densidad y el calor específico de cada uno de los componentes: proteína, grasa, carbohidratos, fibra, ceniza y agua (o humedad). También, se incluye en la parte inferior un consolidado de los cálculos de las propiedades térmicas del alimento que se está analizando.

La clase PropiedadesTermicasGUI, que implementa la interfaz gráfica de usuario, es una subclase de JFrame, y está compuesta por dos ejemplares de la clase de objetos denominada JPanel, donde un ejemplar está referenciado por jPanelEntrada, el cual incluye las etiquetas, cuadro de texto y un botón que le permiten al usuario ingresar los datos de entrada, y el otro ejemplar está referenciado por JPanelSalida, en el que se incrustan los elementos de interfaz gráfica pertinentes para mostrar los resultados de los cálculos. 


\begin{tabular}{|c|}
\hline ComputosPropiedadesTermicas \\
\hline $\begin{array}{l}\text { public int PROTEINA }=0 \\
\text { public int GRASA }=1 \\
\text { public int CARBOHIDRATO }=2 \\
\text { public int FIBRA }=3 \\
\text { public int CENIZA }=4\end{array}$ \\
\hline 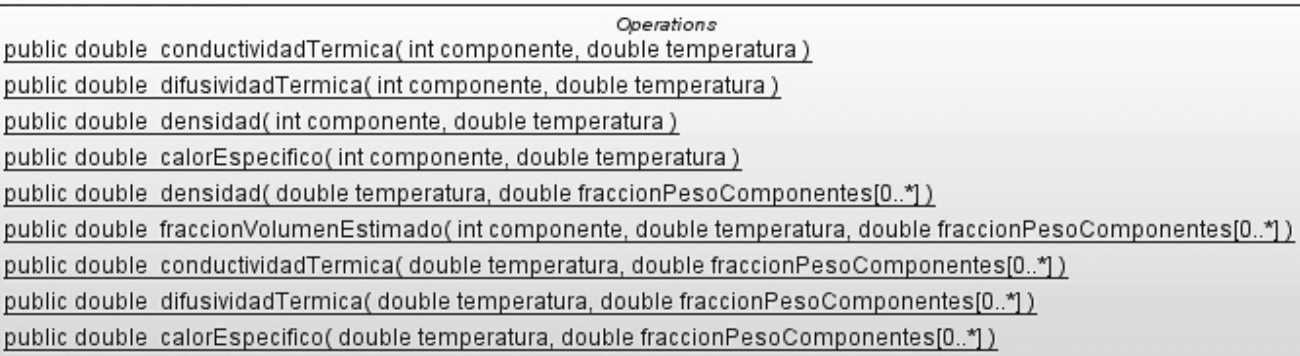 \\
\hline
\end{tabular}

Fig. 2: Diagrama de la clase denominada ComputosPropiedadesTermicas, que contiene los métodos que calculan las propiedades termofísicas, con los modelos de por Choi y Okos (1985)

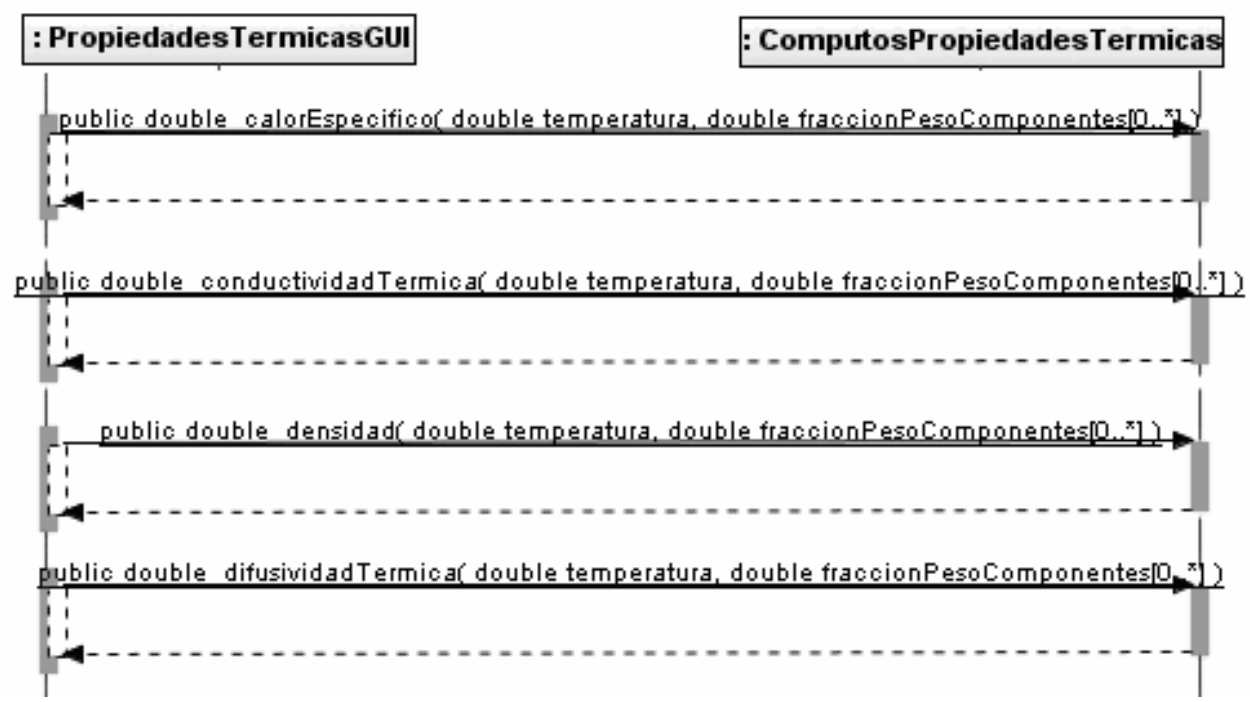

Fig. 3: Diagrama de secuencia que muestra el intercambio de mensajes entre objetos de las clases Propiedades TermicasGUI y ComputosPropiedadesTermicas

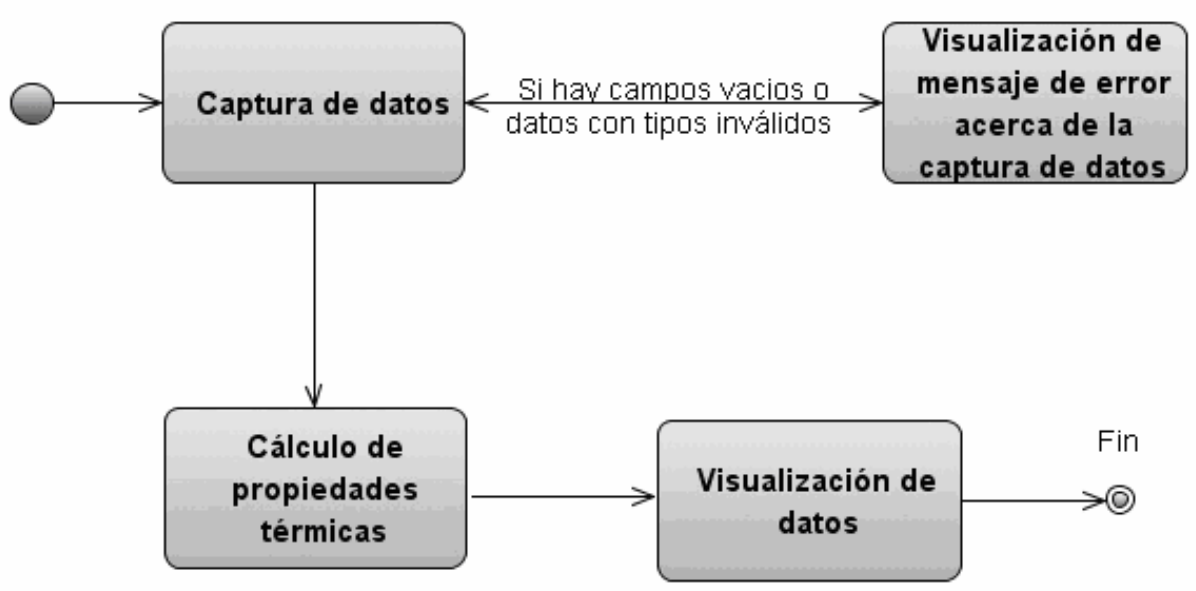

Fig. 4: Diagrama de estados 


\section{ANALISIS DE RESULTADOS}

El programa en ejecución se aprecia en la figura 5, el cual se probó con un trozo de ñame, cuya fracción de peso de proteína, grasa, carbohidratos, fibra, ceniza y humedad, datos experimentales tomados de Alvis (2008), era de 1.84, 7.66, 36.49, 0.096, 1.07 y 52.844 respectivamente, con una temperatura de 140 grados centígrados. Después de pinchar el botón etiquetado como "Calcular propiedades térmicas", el programa de computadoras arrojó que la conductividad térmica, la densidad, la difusividad térmica y el calor específico corresponden con los valores de 0.495 $W / \mathrm{m}^{\circ} \mathrm{C}, 1,45 \times 10^{-7} \mathrm{~m}^{2} / \mathrm{s} 1172.19 \mathrm{~kg} / \mathrm{m}^{3}, 0.145$ y $3.095 \mathrm{~kJ} / \mathrm{kg}^{\circ} \mathrm{C}$.

Las metodologías empleadas en el proyecto fueron: La orientada a objetos en la programación, y la cascada en la construcción de software, la cual tiene cinco etapas descritas por (Booch, 2004; Pressman, 2005): 1) Comunicación: En esta etapa inició el proyecto con la recolección de requisitos funcionales y no funcionales; 2) Planeación: Después de sistematizar los requisitos, se estimó el tiempo empleado para construir el software; 3) Modelado: Después de la planeación, se hizo un análisis y modelado de la solución mediante el paradigma orientado a objetos, en el que se elaboraron las clases de objetos previamente explicados y presentes las figuras 2 y 3 (Rumbaugh et al., 2000; Gomaa, 2004; Pilone et al., 2005; Hamilton et al., 2006; Alvis et al., 2010); 4) Construcción: Una vez terminado el diseño, se codificó una aplicación en el lenguaje de programación denominado Java, y se probó cada requisito funcional una vez implementado. 5) Despliegue: Finalmente, el software fue empaquetado en un fichero de extensión jar e instalado para la enseñanza de propiedades térmicas de los alimentos.

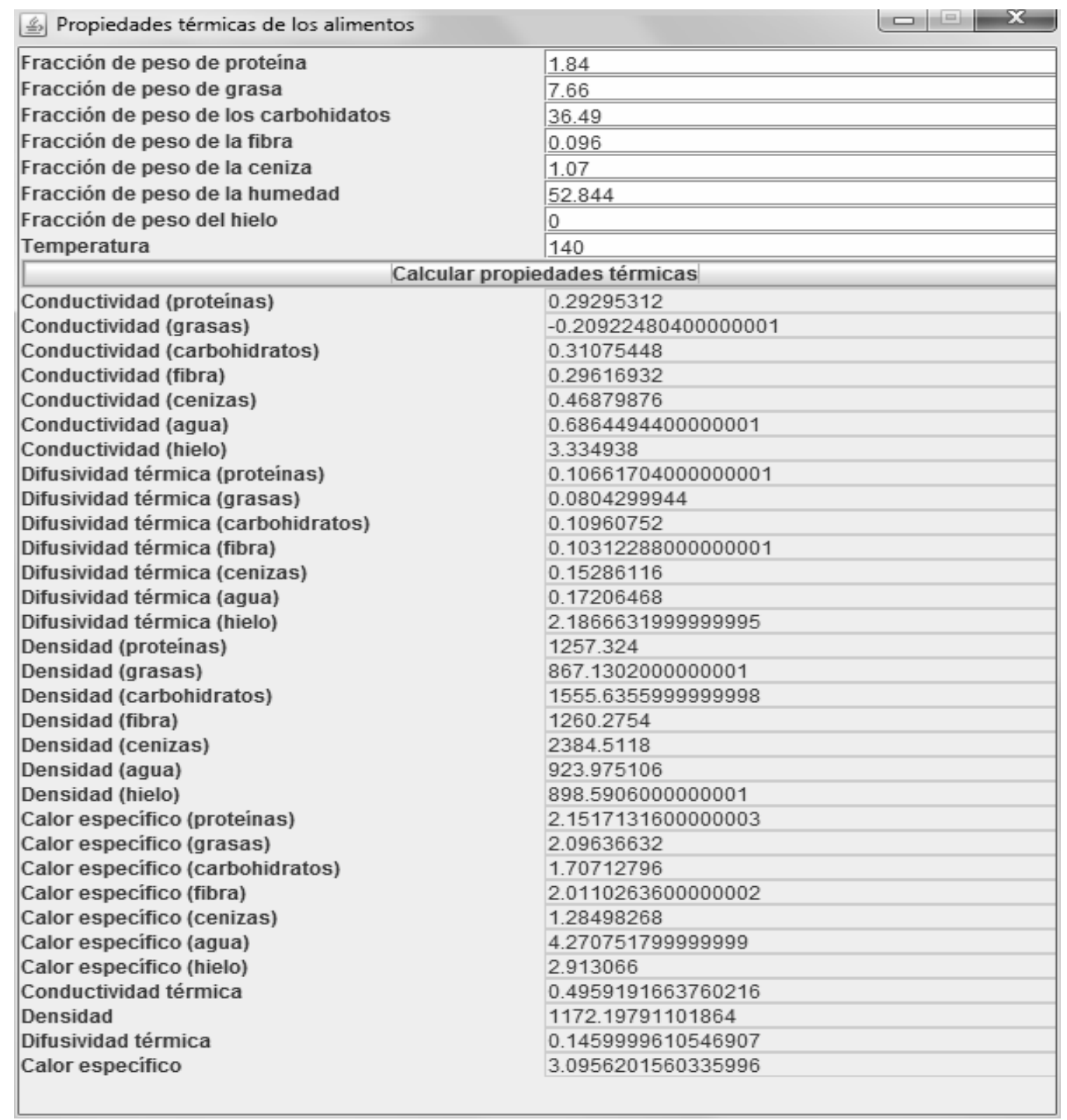

Fig. 5: Programa en ejecución 


\section{CONCLUSIONES}

El presente estudio revela que las aplicaciones computarizadas facilitan la investigación y comprensión de fenómenos ingenieriles concernientes a las propiedades termofísicas en fresco y en procesos térmicos aplicados a la ingeniería de alimentos; partiendo de una serie de datos, los cuales son procesados automáticamente por un programa de computadora elaborado con base en un modelo matemático propuesto.

\section{REFERENCIAS}

Alvis, A., I. Caicedo y P. Peña; Determinación del coeficiente de transferencia de calor a través de una aplicación de computadoras. Información Tecnológica 21(5), 13-20(2010).

Alvis, A., L. Cortés y M. Páez; Transferencia de calor y materia durante la fritura de trozos de ñame (Dioscórea alata). Información Tecnológica 20(1), 99-109(2009).

Alvis, A., C. Vélez y M. Rada-Mendoza; Composición de ñames frescos cultivados en Colombia y sometidos a freído por inmersión. Información Tecnológica 19(1), 3-10(2008).

Barbosa-Canovas, G.V., L. Ma y B. Braletta; Food Engineering Laboratory Manual. Technomic Publishing Company, Inc Lancaster, P.A., (1997).

Bon, J., J. Benedito, J. Cárcel, y A. Mulet; Transferencia de calor en geometría cilindrica considerando resistencia externa: método analítico. Herramientas de cálculo en Ingeniería de Alimentos, p 5-13 (2001).

Booch, G., Rumbaugh, J., Jacobson, I.; El lenguaje unificado de modelado; Editorial Pearson, España, p 28 - 29 (2004).

Califano, A.N. y A. Cálvelo.; Heat and mass transfer during the warn water blanching of potatoes. Journal of food Science. 48(3), 220-225 (1983).

Choi, Y. y M.R. Okos; Effects of temperature and composition on the thermal properties of foodsReview. In Physical and Chemical properties of food. Ed. M. Okos. American Society of Agricultural Engineering, p 93-101(1985)

Gomaa, H.; Designing Software Products Lines with UML: From Use Cases to Pattern - Based

Software Architecture; Editorial Addison Wesley, United States of America (2004).

Hamilton, K., Miles R.; Learning UML 2.0; Editorial O'Reilly, United States of America (2006).

Moreira, R. G; J.K., Palau, VE. Sweat y X. Sun; Thermal and physical properties of tortilla chips as a function of frying time. Journal of Food Processing and Preservation 19(4), 175-189 (1995).

Pelegrina, A.H., R.E. Echarte, y G.H. Sherwood; Cálculo de las propiedades de aire húmedo y su aplicación en la simulación de procesos, p.p 7-23., en Mulet, A., C. Ordorica, J. Benedito y J. MartinezMonzó, Herramientas de cálculo en Ingeniería de Alimentos, V. VI. Universidad Politécnica de Valencia. Valencia. (2000).

Pilone, D., Pitman N.; UML in a Nutshell; Editorial O'Reilly, United States of America (2005).

Pressman, R.; Ingeniería de software, un enfoque práctico; $6^{a}$ Edición, Editorial Mc Graw Hill, México, $p$ 50 - 51 (2005).

Schwartzberg, H. G y R. Y. Chao; Solute diffusivities in leaching process. Food Technology 3(2), 7386(1982).

Rahman, S.; Food properties handbook. ((p. 34)239). Boca Ratón, FL; CRC Press. (1995).

Singh, R.P.; Moving boundaries in food engineering, Journal Food Technology 54(2), 44-53(2000).

Singh, R.P. y F. Erdogdu; Virtual experiments in food processing, RAR Press. Davis, California, (2004).

Rumbaugh, J., Jacobson, I., Booch, G.; El lenguaje unificado de modelado, manual de referencia;

Editorial Pearson, España, p 37 (2000). 